\title{
CZT cameras: A technological jump for myocardial perfusion SPECT
}

\author{
Laetitia Imbert, PhD, ${ }^{\mathrm{a}, \mathrm{b}, \mathrm{c}, \mathrm{d}}$ and Pierre-Yves Marie, $\mathrm{MD}, \mathrm{PhD}^{\mathrm{c}, \mathrm{d}, \mathrm{e}, \mathrm{f}}$ \\ a CRAN, UMR 7039, Université de Lorraine - CNRS, Vandoeuvre, France \\ b Department of Radiotherapy, Institut de Cancérologie de Lorraine, Vandoeuvre, France \\ c Nancyclotep Experimental Imaging Platform, Nancy, France \\ d Department of Nuclear Medicine, CHU Nancy, Nancy, France \\ e Faculty of Medicine, University of Lorraine, Nancy, France \\ ${ }^{f}$ INSERM, U1116, Nancy, France
}

Received Jun 10, 2015; accepted Jun 10, 2015

doi: $10.1007 /$ s $12350-015-0216-2$

\section{See related article, pp. 885-893}

In the past few years, myocardial perfusion imaging has significantly evolved with the development of semiconductor cadmium-zinc-telluride (CZT) cameras, leading to a technological jump in this setting. The hopes raised by this new technology have been substantial because of the potential to improve energy resolution and image quality, compared to conventional Anger cameras. ${ }^{1,2}$ Unlike the latter cameras, these CZT cameras use a principle of direct detection where the interaction of a gamma photon of $140 \mathrm{keV}$ produces approximately 30,000 electrons, a level 20 -fold higher than that produced by a sparkling $\mathrm{NaI}(\mathrm{Tl})$ crystal. ${ }^{3}$ One of the most significant consequences is that energy resolution is improved by a factor of 2 , compared with conventional Anger cameras.

However, the new CZT cameras were also designed to optimize the acquisition and reconstruction processes with original software and collimation systems, leading to a marked enhancement in count sensitivity and spatial resolution, as confirmed by the article by Zoccarato et $\mathrm{al}^{4}{ }^{4}$ in this issue of the Journal of Nuclear Cardiology.

Two semiconductor cameras are currently commercialized: the Discovery NM530c camera (GE Healthcare, Haifa, Israel) and the D.SPECT camera

\footnotetext{
Reprint requests: Laetitia Imbert, PhD, CRAN, UMR 7039, Université de Lorraine - CNRS, 54500 Vandoeuvre, France; l.imbert@nancy. unicancer.fr

J Nucl Cardiol 2016;23:894-6.

$1071-3581 / \$ 34.00$

Copyright (C) 2015 American Society of Nuclear Cardiology.
}

(Spectrum Dynamics, Caesarea, Israel). Both have a limited field of view, due to the substantial cost of semiconductors, and are thus mainly dedicated to cardiology applications. ${ }^{5,6}$ These two cameras use the same type of squared CZT-pixel detector with a surface area of $2.5 \times 2.5 \mathrm{~mm}^{2}$ and a thickness of $5 \mathrm{~mm}$. Consequently, the energy resolution is the same for both cameras and much better than that of conventional cameras. These CZT detectors provide a dramatic improvement in image quality and, in addition, facilitate dual isotope recordings such as those involving ${ }^{201} \mathrm{Tl}$ and ${ }^{99 \mathrm{~m}}$ Tc-labeled tracers. ${ }^{7,8}$

However, the two CZT cameras are based on clearly different collimation systems. This constitutes the main reason why the characteristics of the myocardial perfusion images provided by these two cameras are significantly different, as confirmed by the study of Zoccarato et al. ${ }^{4}$

The Discovery NM530c camera is based on a stationary multi-pinhole collimation system. Each pinhole has an effective aperture diameter of only $5.1 \mathrm{~mm}$ that is likely to advantage spatial resolution and to disadvantage count sensitivity. However, a high count sensitivity may be achieved in this instance, by the simultaneous recording of the heart area through the 19 pinhole-detector blocks. ${ }^{9}$

The D.SPECT camera uses a very different system of rotational parallel-hole collimation. It involves 9 rotating columns associated with a wide-angle squarehole tungsten collimator. This collimator is likely to advantage count sensitivity although a high level of spatial resolution can be reached owing to an original reconstruction method. ${ }^{10}$

In addition to energy resolution, the main parameters allowing to characterize a gamma camera are (1) 
count sensitivity, (2) spatial resolution, and (3) image contrast assessed as a function of noise level (contrast/ noise ratio).

Tomographic count sensitivity is a critical physical parameter on which depends the recording time and the activity that needs to be injected. Thanks to the high sensitivities of CZT cameras, it has been possible to decrease recording times by a factor of 2 whereas the injected activities have decreased up to a factor of 3, leading to dramatic improvements in terms of patient tolerance and radiation doses. ${ }^{11}$

This tomographic count sensitivity is currently being determined on reconstructed images from cardiac phantoms or from a punctual source according to the ratio of recording counts over actual activity. In patients, the actual left ventricular activities are unknown but may be replaced in the formula by the activities which are injected intravenously. ${ }^{12}$ The resulting level of the corresponding parameter is hence around 100-fold lower than that of the actual sensitivity. ${ }^{13}$

Among all commercially available gamma-cameras, the D.SPECT CZT camera is the model showing the highest tomographic count sensitivity, although no more than $1 \%$ of the emitted photons are actually recorded by this camera on cardiac phantoms. This percentage is twofold lower for the Discovery CZT camera, and 7- to 8-fold lower for conventional Anger cameras. ${ }^{12}$ It should be emphasized that these differences are unrelated to the CZT material, although the quality of this detection is likely to be higher with CZT detectors, with a lower proportion of scatter photons. It is indeed the heart-centric methods of collimation of the two CZT cameras that likely explain most of their enhanced count sensitivity when compared with conventional SPECT. ${ }^{14,15}$ In this setting, the wide-angle parallel holes system of the D.SPECT camera is even more gainful when compared with the multi-pinhole system of the Discovery NM 530c.

Spatial resolution is a second major performance parameter. It governs image quality and especially the sharpness of myocardial borders and the detectability of perfusion defects. Spatial resolution is moreover a main determinant of image contrast, the third major performance parameter. However, the high noise level of the SPECT images likely interferes with the detectability of SPECT defects, independently of the estimated contrast values. This is why a contrast-to-noise ratio is currently used for SPECT imaging. It reflects the ability to generate an image contrast which is not impacted by noise fluctuations. $^{12}$

These interdependent parameters of spatial resolution and image contrast reflect "image quality" and in all previously published studies, they were consistently found to be markedly higher on CZT than on conventional Anger cameras, on both phantom ${ }^{14,15}$ and human images. ${ }^{12}$ In addition, these parameters may be slightly higher with the Discovery camera than with the D.SPECT, taking advantage of the pinhole collimation system. This aspect is, however, not as straightforward when analyzing the data documented herein by Zoccarato et al. ${ }^{4}$ The authors used an anthropomorphic torso phantom with a cardiac insert which mimics clinical conditions except for breathing and cardiac motions. The performance parameters could then be assessed with variable count statistics and for the Discovery NM 530c camera, the impact of attenuation correction could also be analyzed. One of the major findings was that the D.SPECT camera exhibited thinner left ventricle walls and an equivalent myocardial/cavity contrast, compared to the Discovery NM 530c camera. This could lead to consider that image quality of the D.SPECT camera is at least equivalent to that of the Discovery camera.

However, contrary to tomographic count sensitivity, these contrast and spatial resolution parameters are highly dependent on the reconstruction parameters. This is well illustrated by the observation that the D.SPECT camera is able to provide a higher level of spatial resolution than conventional Anger cameras, in spite of a wide-hole collimation system and owing to a specific reconstruction algorithm, taking into account the particularities of this collimator.

Routine reconstruction parameters were used in the study of Zoccarato et al, ${ }^{4}$ although it should be kept in mind that the choice of other reconstruction parameters could have impacted their results. Indeed, according to the choice of reconstruction parameters, there is a balance between reconstructed noise on the one hand, and contrast and spatial resolution on the other.

The D.SPECT camera was found in this instance to exhibit thinner left ventricle walls, compared with the Discovery NM 530c camera, a property that may be considered as more favorable in terms of image quality and for facilitating image interpretation. However, these thinner walls are also the consequence of a particular reconstruction system involving a cardiac model. Therefore, they may be considered to not solely reflect a high level of spatial resolution.

There is a relative consensus regarding the fact that the pinhole system of the Discovery camera enables to reach a slightly higher spatial resolution than the D.SPECT camera when conventionally determined on punctual sources, ${ }^{15}$ as well as when assessed by the sharpness of the left ventricular images from patients or phantoms. ${ }^{12}$ It is however very unlikely that such small differences may have a significant impact on diagnostic accuracy. Previous clinical studies have indeed shown that diagnostic accuracy was high for both cameras., 9,10 However, the multi-pinholes system of the Discovery 
camera provides a twofold lower count sensitivity when compared with the D.SPECT camera, and this observation can therefore have a significant impact on recording times and injected activities. In this setting, an additional interesting finding of the study of Zoccarato et $\mathrm{al}^{4}$ is that the image quality parameters remained high for a low level of left ventricular counts (around 500 kcounts). This finding could lead to further reduce the injected activities. ${ }^{11,16}$

In conclusion, the anthropomorphic phantom study of Zoccarato et $\mathrm{al}^{4}$ yields definite evidence that the physical performances of these 2 CZT cameras are relatively similar, even if the corresponding images of the left ventricle are significantly different with a smaller wall thickness for the D.SPECT camera when using routine reconstruction parameters. These cameras are equipped with the same CZT material but operate with different collimation systems and reconstruction algorithms. Finally, it is unlikely, however, that such differences have a significant impact on diagnostic accuracy.

\section{References}

1. Patton JA, Slomka PJ, Germano G, Berman DS. Recent technologic advances in nuclear cardiology. J Nucl Cardiol 2007;14:50113.

2. Slomka PJ, Patton JA, Berman DS, Germano G. Advances in technical aspects of myocardial perfusion SPECT imaging. J Nucl Cardiol 2009; 16:255-76.

3. Nichols KJ, Van Tosh A, Palestro CJ. Prospects for advancing nuclear cardiology by means of new detector designs. J Nucl Cardiol 2009;16:691-6.

4. Zoccarato O, Lizio D, Savi A, Indovina L, Scabbio C, Leva L, et al. Comparative analysis of cadmium zincum telluride cameras dedicated to myocardial perfusion SPECT. A phantom study. J Nucl Cardiol 2015. doi:10.1007/s12350-015-0203-7.

5. Esteves FP, Raggi P, Folks RD, Keidar A, Askew W, Rispler S, et al. Novel solid-state-detector dedicated cardiac camera for fast myocardial perfusion imaging: Multicenter comparison with standard dual detector cameras. J Nucl Cardiol 2009;16:927-34.
6. Sharir T, Ben-Haim S, Merzon K, Prochorov V, Dickman D, BenHaim S, et al. High-speed myocardial perfusion imaging initial clinical comparison with conventional dual detector Anger camera imaging. JACC Cardiovasc Imaging 2008;1:156-63.

7. Berman DS, Kang X, Tamarappoo B, Wolak A, Hayes SW, Nakazato R, et al. Stress thallium-201/rest technetium-99m sequential dual isotope high-speed myocardial perfusion imaging. JACC Cardiovasc Imaging 2009;2:273-82.

8. Ben-Haim S, Kacperski K, Hain S, Van Gramberg D, Hutton BF, Erlandsson K, et al. Simultaneous dual-radionuclide myocardial perfusion imaging with a solid-state dedicated cardiac camera. Eur J Nucl Med Mol Imaging 2010;37:1710-21.

9. Buechel RR, Herzog BA, Husmann L, Burger IA, Pazhenkottil AP, Treyer V, et al. Ultrafast nuclear myocardial perfusion imaging on a new gamma camera with semiconductor detector technique: First clinical validation. Eur J Nucl Med Mol Imaging 2010;37:773-8.

10. Gambhir SS, Berman DS, Ziffer J, Nagler M, Sandler M, Patton J, et al. A novel high-sensitivity rapid-acquisition single-photon cardiac imaging camera. J Nucl Med 2009;50:635-43.

11. Perrin M, Djaballah W, Moulin F, Claudin M, Veran N, Imbert L, et al. Stress-first protocol for myocardial perfusion SPECT imaging with semiconductor cameras: High diagnostic performances with significant reduction in patient radiation doses. Eur $\mathrm{J}$ Nucl Med Mol Imaging 2015;42:1004-11.

12. Imbert L, Poussier S, Franken PR, Songy B, Verger A, Morel O, et al. Compared performance of high-sensitivity cameras dedicated to myocardial perfusion SPECT: A comprehensive analysis of phantom and human images. J Nucl Med 2012;53:1897-903.

13. Verger A, Djaballah W, Fourquet N, Rouzet F, Koehl G, Imbert L, et al. Comparison between stress myocardial perfusion SPECT recorded with cadmium-zinc-telluride and Anger cameras in various study protocols. Eur J Nucl Med Mol Imaging 2013;40:33140.

14. Erlandsson K, Kacperski K, Van Gramberg D, Hutton BF. Performance evaluation of D-SPECT: A novel SPECT system for nuclear cardiology. Phys Med Biol 2009;54:2635-49.

15. Bocher M, Blevis IM, Tsukerman L, Shrem Y, Kovalski G, Volokh L. A fast cardiac gamma camera with dynamic SPECT capabilities: Design, system validation and future potential. Eur J Nucl Med Mol Imaging 2010;37:1887-902.

16. Duvall WL, Croft LB, Ginsberg ES, Einstein AJ, Guma KA, George $\mathrm{T}$, et al. Reduced isotope dose and imaging time with a high-efficiency CZT SPECT camera. J Nucl Cardiol 2011;18:84757. 